\title{
Regulatory Recommendations for Calculating the Unbound Maximum Hepatic Inlet Concentration: A Complicated Story with a Surprising and Happy Ending
}

\author{
Andrew Parkinson \\ XPD Consulting, Shawnee, Kansas
}

Received February 26, 2019; accepted April 2, 2019

The European Medicines Agency (EMA), the US Food and Drug Administration (FDA), and the Japanese Pharmaceutical and Medical Devices Agency (PMDA) all recommend interpreting the results of in vitro studies of cytochrome P450 (P450) inhibition, transporter inhibition, and $\mathrm{P} 450$ induction based on equations that incorporate a relevant in vivo concentration of test drug (also known as the investigational drug, new chemical or molecular entity, inhibitor, inducer, perpetrator, or precipitant) (http://www.ema.europa.eu/docs/ en_GB/document_library/Scientific_guideline/2012/07/WC500129606.pdf; http://www.fda.gov/downloads/Drugs/DevelopmentApprovalProcess/ DevelopmentResources/DrugInteractionsLabeling/UCM237160.pdf; https://www.pmda.go.jp/files/000228122.pdf). For the so-called basic static models of drug interactions, the three main in vivo concentrations are $I_{g u t}, I_{\max , u}$, and $I_{i n, \max , u}$, each of which is used with a safety factor to minimize false-negative predictions. For interactions with intestinal CYP3A and efflux transporters, the relevant in vivo concentration is $I_{g u t}$, the intestinal concentration of an orally administered test drug, which all agencies recommend calculating from dose $/ 250 \mathrm{ml}$. For interactions with hepatic P450 enzymes (both inhibition and induction), hepatic efflux transporters, and renal uptake and efflux transporters, the relevant in vivo concentration is $I_{\text {max }, u}$, the unbound maximum concentration of test drug in plasma after dosing to steady state with the highest intended clinical dose, which all agencies recommend calculating from plasma $C_{\text {max }, \text { ss }} \times f u_{P}$, where $f u_{P}$ is the fraction of unbound drug in plasma with a lower limit of 0.01 (for the basic static models). For interactions with hepatic uptake transporters, such as organic anion transporting peptide (OATP)1B1, OATP1B3, and OCT1, the relevant in vivo concentration is $I_{\text {in, max }, u}$, the unbound maximum hepatic inlet concentration of an orally administered test drug. Here the agencies diverge. In this letter, I point out some potential sources of confusion stemming from differences in how regulatory agencies recommend calculating $I_{\text {in,max }, u}$. It ends on a happy note.

To dispense with the easiest difference right away: The unbound maximum hepatic inlet concentration is called $I_{\text {in,max, }}$ by the FDA, $I_{\text {inlet,max }, u}$ by the PMDA, and $[I]_{u, \text { inlet,max }}$ by the EMA. The first potential source of confusion is that, in the case of the FDA, the term $I_{i n, \max , u}$ refers to the unbound maximum hepatic inlet concentration of test drug in plasma; however, the EMA's term, $[I]_{u, \text { inlet,max }}$, and the PMDA's term, $I_{\text {inlet,max }, u}$, refer to the corresponding concentration in blood. How did these differences arise, and, perhaps more importantly, what impact do they have on interpreting the results of in vitro drug interaction studies? Is it possible that a negative result according to one agency might be positive according to another agency?
All three agencies base their equation for estimating the hepatic inlet concentration of an orally administered drug on a method described by Ito et al. (1998). The original equation was developed for the concentration of test drug in blood (which is evident from Fig. 4 in Ito's article). The equation developed by Ito et al. (1998) is described in eq. 1 because the corresponding equation described by all three agencies contains ambiguities (at best) or errors (at worst).

Based on Ito et al. (1998), the total (bound + unbound) maximum hepatic inlet concentration of a test drug in blood $\left(I_{\text {in, } \max , B}\right)$ can be estimated from the sum of two blood concentrations, namely, the maximum concentration of drug in systemic blood $\left(I_{\max , B}\right.$, also known as $C_{\max , \mathrm{B}}$ ), and the maximum concentration of drug that was absorbed from the gut into hepatic portal blood, which is estimated from four parameters: 1) oral dose; 2) $F_{a}$, the fraction of parent drug absorbed from the gastrointestinal tract into hepatic portal blood; 3 ) the absorption rate constant, $k_{a}$ (the fraction of dose absorbed per unit time); and 4) hepatic blood flow, $Q_{\mathrm{H}}$ (or $\left.Q_{\mathrm{h}}\right)$.

$$
I_{i n, \max , B}=I_{\max , B}+\left(\frac{\text { Dose } \cdot F_{a} \cdot k_{a}}{Q_{H}}\right)
$$

Equation 1 is an abbreviated version of eq. 22 in Ito et al. (1998). The subscript $B$ was added to clarify that the concentrations of test drug are those in blood, not plasma.

In eq. 1 , the term on the left, namely, $I_{\max , B}$, represents drug entering the liver from the systemic circulation, whereas the term on the right, namely (Dose $\left.\cdot F_{a} \cdot k_{a}\right) / Q_{\mathrm{H}}$, represents drug entering the liver from the gut via the hepatic portal vein. Ito et al. (1998) defined $F_{a}$ as the fraction of oral dose that reaches the hepatic portal vein. For the basic static model, all three regulatory agencies replaced Ito's term $F_{a}$ with a new term, namely, $F_{a} \cdot F_{g}$. The agencies redefined $F_{a}$ to mean the fraction of drug that is absorbed from the lumen of the gut, and they defined $F_{g}$ as the fraction of drug that passes through the gut wall into the hepatic portal vein. Accordingly, Ito's term $F_{a}$ is identical to the term $F_{a} \cdot F_{g}$ used by regulatory agencies. The advantage of the term $F_{a} \cdot F_{g}$ is that it captures additional mechanistic information. For example, if the value of $F_{a} \cdot F_{g}$ is 0.5 , it could reflect the situation where $100 \%$ of the drug is absorbed from the gut $\left(F_{a}=1\right)$, but only $50 \%$ reaches the hepatic portal blood $\left(F_{g}=0.5\right)$, perhaps owing to intestinal metabolism, or it could reflect the situation where only half the drug is absorbed from the gut $\left(F_{a}=0.5\right)$, perhaps owing to poor aqueous solubility or efflux by intestinal transporters; but all the drug that is absorbed reaches the hepatic portal blood $\left(F_{g}=1.0\right)$. Accordingly, to reflect the new terms used by regulatory agencies, Ito's equation can be modified as follows in eq. 2 :

$$
I_{i n, \max , B}=I_{\max , B}+\left(\frac{\operatorname{Dose} \cdot F_{a} \cdot F_{g} \cdot k_{a}}{Q_{H}}\right),
$$


where $k_{a}$ is the rate of intestinal absorption $\left(\mathrm{time}^{-1}\right), F_{a}$ is the fraction of dose absorbed from the gut lumen, $F_{g}$ is the fraction of drug that passes through the intestinal wall into blood, and $Q_{H}$ is hepatic blood flow. Unless it is determined experimentally, $k_{a}$ is assumed to have a value of 0.1 minute $^{-1}$, meaning that 0.1 of the dose (i.e., $10 \%$ ) is absorbed every minute. $Q_{H}$, hepatic blood flow, is $97 \mathrm{l} / \mathrm{h}$ for a $70-\mathrm{kg}$ person (the value used by all three regulatory agencies), but in eq. 2 , this is expressed on a per-minute basis to match the units of $k_{a}$; hence, $Q_{\mathrm{H}}=1.62 \mathrm{l} / \mathrm{min}$. If blood concentrations are expressed in micromolar (to match values of $K_{i}$ or $\mathrm{IC}_{50}$ ), then Dose is expressed in micromoles provided $Q_{H}$ is expressed in liters per unit time (i.e., $1.62 \mathrm{l} / \mathrm{min}$ ). The term $F_{a} \cdot F_{g}$ represents the fraction of drug that is absorbed from the intestine into hepatic portal blood. Unless it is determined experimentally, $F_{a} \cdot F_{g}$ is assumed to have a value of 1 , which assumes that all the orally administered parent drug is absorbed from intestine $\left(F_{a}=1\right)$ and all of it crosses the intestinal lining and enters hepatic portal blood $\left(F_{g}=1\right)$.

$R_{b}$ is the ratio of drug concentration in blood $\left(C_{\mathrm{B}}\right)$ to drug concentration in plasma $\left(C_{\mathrm{P}}\right)$; hence $R_{b}=C_{\mathrm{B}} / C_{\mathrm{P}}$. Accordingly, $C_{\mathrm{P}}=$ $\mathrm{C}_{\mathrm{B}} / R_{b}$, and $C_{\mathrm{B}}=C_{\mathrm{P}} \cdot R_{b}$. In other words, $R_{b}$ can be used to convert blood concentrations to plasma concentrations, and vice versa. Dividing all sides of eq. 2 by $R_{b}$ gives eq. 3 :

$$
\frac{I_{i n, \max , B}}{R_{b}}=\frac{I_{\max , B}}{R_{b}}+\frac{\left(\frac{\text { Dose } \cdot F_{a} \cdot F_{g} \cdot k_{a}}{Q_{H}}\right)}{R_{b}}
$$

$I_{\text {in,max }, B} / R_{b}$ is the maximum hepatic inlet concentration of total drug in plasma, which is written by the FDA as $I_{\text {in,max }}$ (without a subscripted $P$ to clarify this is a plasma concentration). Likewise, $I_{\max , B} / R_{b}$ is the maximum systemic concentration of total drug in plasma, which is invariably written as $I_{\text {max }}$; it corresponds to plasma $C_{\max }$. Accordingly, eq. 3 can be simplified as follows:

$$
\text { Plasma } I_{\text {in, } \max }=\text { Plasma } I_{\max }+\frac{\left(\frac{\text { Dose } \cdot F_{a} \cdot F_{g} \cdot k_{a}}{Q_{H}}\right)}{R_{b}}
$$

Equation 4 describes how to calculate the total (bound + unbound) maximum hepatic inlet concentration of drug in plasma. The unbound maximum hepatic inlet concentration in plasma, namely, $I_{i n, \max , u}$, is calculated according to eq. 5 :

$$
\text { Plasma } I_{\text {in }, \text { max }, u}=f u_{P}\left(\text { Plasma } I_{\text {max }}+\frac{\left(\frac{\text { Dose } \cdot F_{a} \cdot F_{g} \cdot k_{a}}{Q_{H}}\right)}{R_{b}}\right)
$$

where $f u_{p}$ is the fraction of unbound drug in plasma (with a lower limit of 0.01 for the basic static model according to all three regulatory agencies).

What equations do regulatory agencies recommend for estimating the maximum hepatic inlet concentration of an orally administered drug?

\section{FDA: Total Hepatic Inlet Concentration}

The FDA calculates the maximum hepatic inlet concentration of total (bound + unbound) drug in plasma based on the eq. 6 (as written in Fig. 6 of the 2017 FDA Guidance for Industry), where $I_{i n \text {,max }}$ is the total maximum hepatic inlet concentration in plasma:

$$
I_{\text {in, } \max }=\left(I_{\max }+\left(F_{a} F_{g} x k_{a} x \text { Dose }\right)\right) / Q_{H} / R_{b}
$$

For clarity, an expanded form of this equation is shown in eq. 7:

$$
/ / I_{\text {in, } \max }=\frac{\frac{I_{\max }+\left(k_{a} \cdot \text { Dose } \cdot F_{a} \cdot F_{g}\right)}{Q_{H}}}{R_{b}}
$$

The expanded form of the equation makes it clear that systemic plasma concentration $\left(I_{\text {max }}\right)$ is being divided by $Q_{H}$ and $R_{b}$, which is applicable only to the estimate of the concentration of drug absorbed from the intestine into hepatic portal blood. The linear form of the FDA equation should be written as follows in eq. 8:

$$
I_{\text {in, } \max }=I_{\max }+\left(\left(\left(F_{a} F_{g} x k_{a} x \text { Dose }\right) / Q_{H}\right) / R_{b}\right)
$$

This single line equation is identical to the expanded version shown in eq. 4.

\section{PMDA: Total Hepatic Inlet Concentration}

Between issuing its draft guidelines in September 2017 (PMDA, 2017) and its final guidelines in February 2018 (PMDA, 2018), the PMDA changed its equation for calculating $I_{\text {inlet,max }}$, the agency's term for the maximum hepatic inlet concentration of total (bound + unbound) drug. The equations appear in the footnote to Figs. 2-5 in both documents. The draft version is shown in eq. 9A; the final version in eq. 9B:

$$
\begin{aligned}
& I_{\text {inlet, } \text { max }}=C_{\text {max }}+\left(k_{a} \cdot \text { dose } \cdot F_{a} \cdot F_{g} / Q_{H}\right) \\
& I_{\text {inlet, } \text { max }}=C_{\text {max }}+\left(k_{a} \cdot \text { dose } \cdot F_{a} \cdot F_{g} / Q_{H}\right)
\end{aligned}
$$

The fact the equations are identical conceals an important difference, namely, that, in eq. $9 \mathrm{~A}, C_{\max }$ is defined as the maximum concentration of total drug in plasma, whereas in eq. 9B it is defined as the maximum concentration of total drug in blood. Accordingly, in the draft version, $I_{\text {inlet, max }}$ was calculated as the sum of two concentrations, one in plasma $\left(C_{\max }\right)$ and one in portal blood, such that $I_{\text {inlet, } \max }$ was neither plasma concentration nor blood concentration but a combination of both. By changing the definition of $C_{\max }$ from a plasma concentration to a blood concentration, the final version of the PMDA guidelines corrected this error and made $I_{\text {inlet,max }}$ the maximum hepatic concentration of total drug in blood. For clarity, the PMDA's final equation can be written as follows in eq. $9 \mathrm{C}$ :

$$
\text { Blood } I_{\text {inlet }, \text { max }}=C_{\text {max }, B}+\left(\frac{k_{a} \cdot \text { dose } \cdot F_{a} \cdot F_{g}}{Q_{H}}\right)
$$

\section{EMA: Total Hepatic Inlet Concentration}

The EMA does not describe an equation for calculating the total maximum hepatic inlet concentration, but it does describe an equation for calculating the unbound maximum hepatic inlet concentration in blood, which is described later. Had the EMA described a separate equation for calculating the total maximum hepatic inlet concentration in blood, it would have been the same as eq. 9C (the PMDA's equation).

\section{FDA: Unbound Maximum Hepatic Inlet Concentration in Plasma}

The preceding FDA equation (eq. 4) is intended to calculate the total (bound + unbound) maximum hepatic inlet drug concentration in plasma (called $I_{\text {in,max }}$ by the FDA). The corresponding unbound concentration is calculated as follows: $I_{\text {in, } \max , u}=f u_{P} \cdot I_{i n, \max }$, as shown in eq. 5 . 


\section{EMA and PMDA: Unbound Maximum Hepatic Inlet Concentration in Blood}

The PMDA does not show an expanded equation for calculating the unbound maximum hepatic concentration in blood, but the flowchart in Figs. $2-5$ shows that this is calculated as $f u_{B} \times I_{\text {inlet,max }}$, where $f u_{B}$ is the unbound concentration of drug in blood, and $I_{\text {inlet,max }}$ is the total maximum hepatic inlet concentration in blood, which is calculated as shown in eq. 9C.

The EMA describes an equation for estimating the unbound hepatic inlet concentration of drug in blood, termed [I] $]_{\text {,inlet,max }}$, as follows:

$$
[I]_{u, \text { inlet }, \text { max }}=f u_{B} \cdot\left([I]_{\max , B}+F_{a} \cdot F_{g} \cdot k_{a} \cdot \text { Dose } / Q_{H}\right)
$$

This equation is correct provided the term $F_{a} \cdot F_{g} \cdot k_{a} \cdot$ Dose is divided by $Q_{H}$ before it is added to $[I]_{\max , B}$. Adding brackets around the term $F_{a}$. $F_{g} \cdot k_{a} \cdot D_{0 s e} / Q_{H}$ would remove any ambiguity. Accordingly, the EMA and PMDA both describe an equation (eq. 10) for calculating the unbound maximum hepatic inlet concentration of drug in blood, whereas the FDA describes an equation (eq. 5) for calculating the corresponding concentration in plasma.

The concentration of a circulating test drug is rarely measured in blood; it is nearly always measured in plasma. $[I]_{\max , B}$ can be estimated by multiplying plasma $C_{\max }$ by $R_{b}$. Likewise, protein binding of a test drug is usually measured in plasma, not blood. The unbound fraction of drug in blood $\left(f u_{B}\right)$ can be estimated by dividing the unbound fraction in plasma $\left(f u_{P}\right)$ by $R_{b}$. These relationships are summarized in eq. 11:

$$
C_{B}(\text { blood concentration }) \cdot f u_{B}=C_{P}(\text { plasma concentration }) \cdot f u_{P}
$$

Accordingly, the EMA and PMDA equations can be written as follows in eq. 12 (where the subscripts B and P represent blood and plasma, respectively):

$$
[I]_{u, \text { inlet }, \max , B}=\left(\frac{f u_{P}}{R_{b}}\right) \cdot\left(\left([I]_{\max , P} \cdot R_{b}\right)+\left(\frac{F_{a} \cdot F_{g} \cdot k_{a} \cdot \text { Dose }}{Q_{H}}\right)\right)
$$

\section{Blood Versus Plasma}

How do plasma levels of total maximum hepatic inlet concentration compare with blood levels, and how do plasma values of unbound maximum hepatic inlet concentration compare with blood values? Simulated comparisons were made for drugs with an $R_{b}$ of 0.5 , 1.0, and 2.0. The oral dose of each drug was $325 \mu \mathrm{mol}$. Each drug had a systemic plasma $C_{\max }$ of $4.0 \mu \mathrm{M}$, and each was $80 \%$ bound to plasma protein $\left(f u_{P}=0.2\right)$. In each case, $F_{a} \cdot F_{g}$ was 1 , and $k_{a}$ was 0.1 minute $^{-1}$. Hepatic blood flow $\left(Q_{\mathrm{H}}\right)$ was $1.62 \mathrm{ml} / \mathrm{min}$. Table 1 shows calculations of total (bound + unbound) and unbound maximum hepatic inlet concentration in plasma, as recommended by the FDA, and calculations of the corresponding concentrations in blood, as recommended by the EMA and PMDA. The total maximum hepatic inlet concentrations in plasma (the FDA's approach) differ from those in blood (the EMA's and PMDA's approach), depending on the value of $R_{b}$; however, the values of unbound maximum hepatic inlet concentration are identical in plasma and blood for a given value of $R_{b}$. This is because to convert plasma concentration to blood concentration, you multiply by $R_{b}\left(C_{\mathrm{B}}=C_{\mathrm{P}} \times R_{b}\right)$; but to convert the unbound fraction of drug in plasma to unbound concentration in blood, you divide by $R_{b}\left(f u_{B}=f u_{P} / R_{b}\right)$, based on the relationship shown in eq. 11 . The two cancel each other out.
A formal proof that the EMA/PMDA and FDA's equations for calculating the unbound maximum hepatic inlet concentration in blood and plasma, respectively, are identical is as follows:

Eq. 12 - the EMA's (and PMDA's) equation-is as follows:

$$
\begin{aligned}
\operatorname{Blood}[I]_{u, \text { inlet }, \max }= & \left(\frac{f u_{P}}{R_{b}}\right) \cdot\left(\left([I]_{\max , P} \cdot R_{b}\right)\right. \\
& \left.+\left(\frac{F_{a} \cdot F_{g} \cdot k_{a} \cdot \text { Dose }}{Q_{H}}\right)\right)
\end{aligned}
$$

Multiplying each of the terms for systemic blood concentration and portal blood concentration by $f u_{p} / R_{b}$ gives the expanded version eq. 13 :

$\operatorname{Blood}[I]_{u, \text { inlet }, \text { max }}=\left(f u_{p} \cdot\left([I]_{\max , P}\right)+\frac{f u_{p}}{R_{b}} \cdot\left(\frac{F_{a} \cdot F_{g} \cdot k_{a} \cdot \text { Dose }}{Q_{H}}\right)\right)$

Factoring out $f u_{p}$ gives the contracted equation:

$$
\operatorname{Blood}[I]_{u, \text { inlet }, \max }=f u_{p} \cdot\left(\left([I]_{\max , P}\right)+\frac{1}{R_{b}} \cdot\left(\frac{F_{a} \cdot F_{g} \cdot k_{a} \cdot \text { Dose }}{Q_{H}}\right)\right)
$$

Eq. 14, a rearrangement of the EMA's equation for calculating the unbound maximum hepatic inlet concentration in blood, is identical to eq. 5 , the FDA's equation for calculating the unbound maximum hepatic inlet concentration in plasma:

$$
\text { Plasma } I_{\text {in }, \text { max }, u}=f u_{P}\left(\text { Plasma } I_{\max }+\frac{\left(\frac{\text { Dose } \cdot F_{a} \cdot F_{g} \cdot k_{a}}{Q_{H}}\right)}{R_{b}}\right)
$$

One point needs to be emphasized. I stated previously that the values of unbound maximum hepatic inlet concentration are identical in blood and plasma for a given value of $R_{b}$. The qualification "for a given value of $R_{b}$ " is important. As shown in Table 1 , values of $R_{b}$ do have an impact on the unbound maximum hepatic inlet concentration, but for a given drug with a given $R_{b}$ value, the unbound maximum hepatic inlet concentration in blood is the same as that in plasma; so it makes no difference whether you calculate the FDA's value of plasma $I_{\text {in, max }, u}$ according to eq. 5 or calculate the EMA's/PMDA's value of blood $[I]_{u, \text { inlet,max }}$ according to eq. 12 .

All this suggests that, when evaluating the inhibition of hepatic uptake transporters based on the unbound maximum hepatic inlet concentration, a negative result obtained with the FDA's approach would also be negative according to the EMA and PMDA's approach. That would be true if the three agencies used the same equations and cutoff criteria, but they do not. The FDA and PMDA's equation and cutoff for inhibition of hepatic uptake transporters (OATP1B1 and OAPT1B3) are shown in eq. 15.

$$
1+\frac{I_{\text {in, } \max , u}}{K_{i} \text { or } I C_{50}} \geq 1.1
$$

The EMA's equation and cutoff for inhibition of hepatic uptake transporters (OATP1B1, OAPT1B3, and OCT1) are shown in eq. 16:

$$
\frac{25 \cdot[I]_{u, \text { inlet }, \max }}{K_{i} \text { or } I C_{50}} \geq 1
$$

The EMA's equation is equivalent to an FDA/PMDA-style eq. $(1+$ ratio) with a cutoff value of $1.04(=1+1 / 25)$, compared with 1.1 for the 
TABLE 1

Total (bound + unbound) and unbound maximum hepatic inlet concentration in plasma (the FDA approach) and blood (the EMA and PMDA approach) for drugs with different $R_{b}$ values

Simulated comparisons were made for drugs $A, B$, and C with an $\mathrm{R}_{\mathrm{b}}$ of $0.5,1.0$, and 2.0, respectively. The oral dose of each drug was $325 \mu$ mol. Each drug had a total (bound + unbound) systemic plasma $C_{\max }$ of $4.0 \mu \mathrm{M}$, and each was $80 \%$ bound to plasma protein $\left(f u_{P}=0.2\right)$. In each case, $F_{a} \cdot F_{g}$ was 1 , and $k_{a}$ was 0.1 min ${ }^{-1}$. Hepatic blood flow $\left(Q_{H}\right)$ was $1.62 \mathrm{l} / \mathrm{min}$.

\begin{tabular}{|c|c|c|c|c|c|c|c|c|c|c|c|}
\hline \multirow[t]{2}{*}{ Drug } & \multirow[t]{2}{*}{$R_{b}$} & \multicolumn{2}{|c|}{ Total Systemic $C_{\max }$} & \multicolumn{2}{|c|}{ Total Portal $C_{\max }$} & \multicolumn{2}{|c|}{$\begin{array}{l}\text { Total Maximum Hepatic Inlet } \\
\text { Concentration }\left(I_{i n, \max }\right)\end{array}$} & \multicolumn{2}{|c|}{ Fraction Unbound } & \multicolumn{2}{|c|}{$\begin{array}{l}\text { Unbound Maximum Hepatic Inlet } \\
\text { Concentration }\left(I_{i n, \max , u}\right)\end{array}$} \\
\hline & & Plasma $^{a}$ & Blood $^{b}$ & Plasma $^{c}$ & Blood $^{d}$ & Plasma $^{e}$ & Blood $^{f}$ & Plasma $^{g}$ & Blood $^{h}$ & Plasma $^{i}$ & Blood $^{j}$ \\
\hline & & $\mu M$ & $\mu M$ & $\mu M$ & $\mu M$ & $\mu M$ & $\mu M$ & $f u_{P}$ & $f u_{B}$ & $\mu M$ & $\mu M$ \\
\hline A & 0.5 & 4 & 2 & 40 & 20 & 44 & 22 & 0.2 & 0.4 & 8.8 & 8.8 \\
\hline B & 1.0 & 4 & 4 & 20 & 20 & 24 & 24 & 0.2 & 0.2 & 4.8 & 4.8 \\
\hline $\mathrm{C}$ & 2.0 & 4 & 8 & 10 & 20 & 14 & 28 & 0.2 & 0.1 & 2.8 & 2.8 \\
\hline \multicolumn{12}{|c|}{ FDA (based on drug concentrations in plasma) } \\
\hline A & 0.5 & 4 & & 40 & & 44 & & 0.2 & & 8.8 & \\
\hline $\mathrm{B}$ & 1.0 & 4 & & 20 & & 24 & & 0.2 & & 4.8 & \\
\hline $\mathrm{C}$ & 2.0 & 4 & & 10 & & 14 & & 0.2 & & 2.8 & \\
\hline \multicolumn{12}{|c|}{ EMA and PMDA (based on drug concentrations in blood) } \\
\hline A & 0.5 & & 2 & & 20 & & 22 & & 0.4 & & 8.8 \\
\hline $\mathrm{B}$ & 1.0 & & 4 & & 20 & & 24 & & 0.2 & & 4.8 \\
\hline $\mathrm{C}$ & 2.0 & & 8 & & 20 & & 28 & & 0.1 & & 2.8 \\
\hline
\end{tabular}

EMA, European Medicines Agency; FDA, US Food and Drug Administration; PMDA, Pharmaceutical and Medical Devices Agency; $R_{\mathrm{b}}$, Ratio of drug concentration in blood $\left(C_{\mathrm{B}}\right)$ to drug concentration in plasma $\left(C_{\mathrm{P}}\right)$.

${ }^{a}$ The total drug concentration in systemic plasma is determined experimentally. It was set to $4 \mu \mathrm{M}$ for drugs A-C.

${ }^{b}$ Calculated as follows: Total systemic $C_{\max }$ in blood $=$ Total systemic $C_{\max }$ inplasma $\cdot R_{b}$.

${ }^{c}$ Calculated as follows: Total portal $C_{\max }$ in plasma $=\frac{\left(\frac{\text { Dose } \times F_{a} \times F_{g} \times k_{a}}{Q_{H}}\right)}{R_{b}}=\frac{\left(\frac{325 \mu \mathrm{mol} \times 1.0 \times 0.1 \mathrm{~min}^{-1}}{1.62 \mathrm{l} / \mathrm{min}}\right)}{R_{b}}$.

${ }^{d}$ Calculated as follows: Total portal $C_{\max }$ in blood $=\frac{\text { Dose } \times F_{a} \times F_{g} \times k_{a}}{Q_{H}}=\frac{325 \mu \mathrm{mol} \times 1.0 \times 0.1 \mathrm{~min}^{-1}}{1.62 \mathrm{l} / \mathrm{min}}$.

${ }^{e}$ Calculated as follows: Total $I_{\text {in,max }}$ in plasma $=$ Total systemic $C_{\max }$ in plasma + Total portal $C_{\max }$ in plasma.

${ }^{f}$ Calculated as follows: Total $I_{\text {in,max }}$ in blood $=$ Total systemic $C_{\max }$ in blood + Total portal $C_{\max }$ in blood,.

${ }^{g}$ The unbound concentration of drug in plasma $\left(f u_{P}\right)$ is determined experimentally. It was set to 0.2 for Drugs A-C.

${ }^{h}$ Calculated as follows: $f u_{B}=\frac{f u_{P}}{R_{b}}$.

${ }^{i}$ Calculated as follows: Plasma $I_{i n, \text { max }, u}=$ Plasma $I_{\text {in }, \max } \times f u_{P}$.

${ }^{j}$ Calculated as follows: Blood $I_{i n, \max , u}=\operatorname{Blood}[I]_{\text {in, } \max } \times f u_{B}$.

FDA and PMDA. In other words, the EMA is more conservative than the other two agencies.

Another important difference is the FDA, which updated its Guidance in 2017, and the PMDA, which updated its guidelines in 2018, now recommend evaluating test drugs as inhibitors of OATPs with and without a 30-minute preincubation of the test drug with the test system to assess the potential for time-dependent inhibition of OATPs.

In the case of the basic static models, the unbound maximum hepatic inlet concentration is used only to evaluate the potential for a test drug to cause clinically relevant inhibition of hepatic uptake transporters (OATP1B1, OATP1B3, and, for the EMA, OCT1). It is also used when drug interactions are assessed by the static mechanistic model the mechanistic static model, or the mechanismbased static pharmacokinetic (MSPK) model, which are all one and the same. This model provides an assessment of the net effect of reversible inhibition, irreversible inhibition, and induction of $\mathrm{P} 450$ enzymes in the liver and intestine. Despite describing this model, the EMA and FDA both recommend that an assessment of P450 induction potential should not be combined with assessments of reversible and irreversible $\mathrm{P} 450$ inhibition potential, but the static mechanistic model can be used to assess the combined impact of reversible and irreversible $\mathrm{P} 450$ inhibition. When the static mechanistic model is used to assess the impact of a test drug on hepatic enzymes, all three agencies recommend basing the assessment on the unbound maximum hepatic inlet concentration, which all three agencies call $[I]_{\mathrm{h}}$. The EMA makes it clear that $\left[I_{\mathrm{h}}\right.$ corresponds to $[I]_{u, \text { inlet,max }}$ (its term for the unbound maximum hepatic inlet concentration), and both are calculated from the same equation (Table 1 in the 2012 EMA Guideline on the Investigation of Drug Interactions). In the body of its updated guidelines, the PMDA does not specify that $[I]_{\mathrm{h}}$ corresponds to $I_{\text {inlet, } \max , u}$ (its term for the

$$
[I]_{u, \text { inlet } \text { max }}=f_{u, b}\left([I]_{\max , b} \downarrow^{\frac{\text { Add a plus sign here }}{\text { Dose } x F_{a} x k_{a}}} Q_{h}\right)
$$

Fig. 1. A published equation for calculating the unbound maximum hepatic inlet concentration in blood that contains an error, namely, a missing plus sign. This equation is eq. 3 from Vieira et al. (2014). [I] $]_{\text {u.nllet, } \max }$ is the unbound maximum hepatic inlet concentration of drug in blood, $f_{u, b}$ is the unbound fraction of drug in blood, $[I]_{\max b}$ is the maximum concentration of total drug in systemic blood, and (Dose $\left.\times F_{a} \times k_{a}\right) / Q_{\mathrm{h}}$ is the maximum concentration of drug in hepatic portal blood. $Q_{\mathrm{h}}$ is the sum of the blood flow in hepatic artery and portal vein $(1.617 \mathrm{l} / \mathrm{h}), k_{a}$ is the rate of absorption of drug from the intestine, and $F_{a}$ is the fraction of drug absorbed from the intestine into portal blood, as originally described by Ito et al. (1998), which all three regulatory agencies have replaced with $F_{a} \cdot F_{g}$, as described in the text. 
TABLE 2

Summary of nomenclature and methods used by regulatory agencies to calculate the unbound maximum hepatic inlet concentration of an orally administered drug

\begin{tabular}{|c|c|c|c|c|}
\hline Agency & $\begin{array}{l}\text { Abbreviated Terms for Total and Unbound } \\
\text { Hepatic Inlet Concentration }\end{array}$ & Matrix & Basic Equation for Calculating Total and Unbound Hepatic Inlet Concentration ${ }^{a, b}$ & Equation in Text \\
\hline FDA & $I_{\text {in, } \max ,} I_{\text {in, max,u }}$ & Plasma & $\begin{array}{l}\text { Total plasma } I_{\text {in,max }}=\text { systemic } C_{\text {max }} \text { in plasma }+ \text { portal } C_{\text {max }} \text { in plasma } \\
\text { Plasma } I_{\text {in,max,u }}=\text { plasma } I_{\text {in,max }} \times f u_{P}\end{array}$ & Eq. 5 \\
\hline EMA & {$[I]_{\text {inlet,max }},[I]_{\mathrm{u}, \text { inlet, } \max }$} & Blood & $\begin{array}{l}\text { Total blood }[I]_{\text {inlet,max }}=\text { systemic } C_{\max } \text { in blood }+ \text { portal } C_{\max } \text { in blood } \\
\text { Blood }[I]_{\mathrm{u}, \text { inlet, } \max }=\text { blood }[I]_{\text {inlet,max }} \times f u_{B}\end{array}$ & Eq. 12 \\
\hline PMDA & $I_{\text {inlet,max }}, I_{\text {inlet,max,u }}$ & & $\begin{array}{l}\text { Total blood } I_{\text {inlet,max }}=\text { systemic } C_{\max } \text { in blood }+ \text { portal } C_{\max } \text { in blood } \\
\text { Blood } I_{\text {inlet,max,u }}=\text { blood } I_{\text {inlet,max }} \times f u_{B}\end{array}$ & \\
\hline
\end{tabular}

EMA, European Medicines Agency; FDA, US Food and Drug Administration; PMDA, Pharmaceutical and Medical Devices Agency.

${ }^{a}$ fup and $f u_{B}$ are the fraction of unbound drug in plasma and blood, respectively.

${ }^{b}$ The only difference between the EMA and PMDA equations is the abbreviated terms used for the total/unbound maximum hepatic inlet concentration.

unbound maximum hepatic inlet concentration), nor does it provide an equation for calculating $[I]_{\mathrm{h}}$. It simply states that $\left[I_{\mathrm{h}}\right.$ is the "concentration of the investigational drug in the liver hepatocytes"; however, the 2018 PDMA guidelines end with a "Question \& Answer" section. In response to question 15, the PMDA clarifies that $[I]$ h corresponds to $I_{\text {inlet,max }, u}$, which the agency recommends calculating from eq. 10, which is the EMA's equation for calculating $[I]_{u, \text { inlet,max }}$. The FDA does not specify that $[I]_{\mathrm{h}}$ corresponds to $I_{\text {in,max,u }}$ (its term for the unbound maximum hepatic inlet concentration), but it does provide an equation for calculating []$_{\mathrm{h}}$ (Fig. 7 in the 2017 FDA Guidance for Industry); however, the equation for calculating $[I]_{\mathrm{h}}$ is not identical to the equation for calculating $I_{\text {in,max }, u}$. For reasons outlined earlier, to calculate $I_{i n, \max , u}$ for the basic static model, the FDA replaced Ito's original term $F_{a}$ with the combined term $F_{a} \cdot F_{g}$ (see eq. 5); however, to calculate $\left[I_{\mathrm{h}}\right.$ for the static mechanistic model, the FDA retained Ito's original term and definition of $F_{a}$ (Fig. 7 in the 2017 FDA Guidance for Industry); hence, $F_{g}$ appears in the equation to calculate $I_{i n, \max , u}$ but not in the equation to calculate $\left[I I_{\mathrm{h}}\right.$, even though they are the same thing, namely, the unbound maximum hepatic inlet concentration. Equation 5 should be used to calculate plasma $I_{\text {in,max }, u}$ and $[I]_{\mathrm{h}}$.

I will touch on three related topics before I close with a summary.

First, the concentration of test drug in hepatic portal blood is estimated from dose, $k_{a}, F_{a} \cdot F_{g}$, and $Q_{\mathrm{H}}$. I do not know why blood flow is based on total hepatic blood flow $(97 \mathrm{l} / \mathrm{h}$ ) and not on hepatic portal blood flow, which is roughly $80 \%$ of total hepatic blood flow (Carlisle et al., 1992).

Second, as a general rule, nonionized and basic drugs tend to distribute evenly between plasma and erythrocytes with $R_{b}$ values close to 1 , whereas zwitterionic and acidic drugs tend to be excluded from erythrocytes with $R_{b}$ values of roughly 0.55 (which is 1-hematocrit) (Hallifax et al., 2010). Some drugs, however, have a high blood-to-plasma concentration ratio. For example, $R_{b}$ values for tacrolimus average about 15 , but the values are concentration-dependent and vary from 4 to 114 (Wallemacq and Verbeeck, 2001). The partitioning of tacrolimus into erythrocytes does not restrict its hepatic clearance, which is due mainly to metabolism by CYP3A. Accordingly, whereas the hepatic clearance of intravenously administered tacrolimus from blood ranges from 2.1 to $6.3 \mathrm{l} / \mathrm{h}$ (for a $70-\mathrm{kg}$ person), which is well below hepatic blood flow $(97 \mathrm{l} / \mathrm{h})$, hepatic clearance from plasma ranges from 42 to $378 \mathrm{l} / \mathrm{h}$, which greatly exceeds hepatic blood flow $(97 \mathrm{l} / \mathrm{h})$, and hepatic plasma flow ( $\sim 53 \mathrm{l} / \mathrm{h}$ ) (Wallemacq and Verbeeck, 2001). The finding that the plasma clearance of tacrolimus $\left(C L_{\mathrm{P}}\right)$ is equal to blood clearance $\left(C L_{\mathrm{B}}\right) \times R_{b}$ suggests that the hepatic clearance of tacrolimus is not restricted by its extensive partitioning into erythrocytes (owing to its binding to an intracellular immunophilin known as FK-binding protein-12 or FKBP12); however, not all drugs with a high $R_{b}$ value behave like tacrolimus. Some diuretic drugs, such as chlorthalidone, dorzolamide, and methazolamide, bind with high affinity to carbonic anhydrase, an abundant enzyme in erythrocytes (Hinderling, 1997). These drugs have $R_{b}$ values ranging from 30 to 240 , but they do not move freely between erythrocytes and plasma; hence, for these drugsdrugs whose movement out of erythrocytes is restricted-it is their concentration in plasma that is relevant to their potential to cause drug interactions (Hinderling, 1997). When a drug has a high value of $R_{b}$, it would be prudent to establish that partitioning into erythrocytes is readily reversible before using $R_{b}$ to interconvert values of drug concentration in blood and plasma, protein binding in blood and plasma, and/or clearance from blood and plasma. Such interconversions are not appropriate when high values of $R_{b}$ reflect restrictive binding of drugs to erythrocytes.

Third, hoping to find some clarification on issues surrounding equations for calculating the unbound maximum hepatic inlet concentration, I turned to a publication authored by representatives from the FDA, EMA, pharma, and academia (Vieira et al., 2014). Their equation for calculating the unbound maximum hepatic inlet concentration in blood (called $[I]_{u, \text { inlet,max }}$ after the EMA) is shown in Fig. 1. I include it here for two reasons. First, on the positive side, the equation reinforces the importance of using blood concentration in equations that include one or more terms for organ blood flow. This same point was made by Yang et al. (2007) for estimating hepatic clearance according the well stirred model, an equation that includes a term for hepatic blood flow. Both publications point to the importance of taking $R_{b}$ into account. On the negative side, the published equation for calculating the unbound maximum hepatic inlet concentration is missing a plus sign, as shown in Fig. 1, which supports my contention that this is a rather tricky area to navigate. It is also worth noting that, for the fraction of drug absorbed from the intestine into hepatic portal blood, the equation shown in Fig. 1 uses $F_{a}$, the term originally used by Ito et al. (1998) rather than $F_{a} \cdot F_{g}$, the term now used by all three regulatory agencies, as discussed earlier.

In summary, the maximum hepatic inlet concentration of a test drug is the sum of two concentrations: systemic $C_{\max }$ and hepatic portal $C_{\max }$. As summarized in Table 2, the FDA bases the maximum hepatic inlet concentration on the sum of these two concentrations in plasma, whereas the EMA and PMDA base it on the sum of these two concentrations in blood. Estimates of the total (bound + unbound) maximum hepatic inlet concentration in plasma differ from those in blood unless $R_{b}$, the blood-to-plasma concentration ratio, is 1 , as shown in Table 1 . However, assessing the potential for a test drug to cause clinically relevant inhibition of hepatic uptake transporters, such as OATP1B1, OATP1B3, and OCT1 (according to the basic static models), or inhibition of hepatic P450 enzymes (according to the static mechanistic models) is based on the unbound maximum hepatic inlet concentration. Surprisingly, for a given value of $R_{b}$, the unbound maximum hepatic inlet concentration in plasma is identical to the unbound maximum hepatic inlet concentration in blood, as shown in Table 1. For FDA submissions, plasma 
$I_{\text {in, } \max , u}$ can be calculated from eq. 5. For EMA and PMDA submissions, blood $[I]_{u, \text { inlet,max }}\left(\right.$ EMA) and blood $I_{\text {inlet,max }, u}$ (PMDA) can be calculated from eq. 12 But, I am happy to say, all these values of unbound maximum hepatic inlet concentration are the same.

\section{References}

Carlisle KM, Halliwell M, Read AE, and Wells PN (1992) Estimation of total hepatic blood flow by duplex ultrasound. Gut 33:92-97.

Hallifax D, Foster JA, and Houston JB (2010) Prediction of human metabolic clearance from in vitro systems: retrospective analysis and prospective view. Pharm Res 27:2150-2161.

Hinderling PH (1997) Red blood cells: a neglected compartment in pharmacokinetics and pharmacodynamics. Pharmacol Rev 49:279-295.

Ito K, Iwatsubo T, Kanamitsu S, Ueda K, Suzuki H, and Sugiyama Y (1998) Prediction of pharmacokinetic alterations caused by drug-drug interactions: metabolic interaction in the liver. Pharmacol Rev 50:387-412.
PMDA (2017). Pharmaceuticals and Medical Devices Agency (Japan): Drug Interaction Guideline for Drug Development and Labelling Recommendations. Draft version issued September 2017. Available in English at: https://www.xenotech.com/scientific resources/regulatory-documents/2017/2017-japanese-pmda-guidance-english.aspx\#.WsJyDbruP

Vieira ML, Kirby B, Ragueneau-Majlessi I, Galetin A, Chien JY, Einolf HJ, Fahmi OA, Fischer V, Fretland A, Grime K, et al. (2014) Evaluation of various static in vitro-in vivo extrapolation models for risk assessment of the CYP3A inhibition potential of an investigational drug. Clin Pharmacol Ther 95:189-198.

Wallemacq PE and Verbeeck RK (2001) Comparative clinical pharmacokinetics of tacrolimus in paediatric and adult patients. Clin Pharmacokinet 40:283-295.

Yang J, Jamei M, Yeo KR, Rostami-Hodjegan A, and Tucker GT (2007) Misuse of the well-stirred model of hepatic drug clearance. Drug Metab Dispos 35:501-502.

Address correspondence to: Dr. Andrew Parkinson, XPD Consulting, 18000 West 68th Street, Shawnee, KS 66217. E-mail: aparkinson@xpd.us.com 